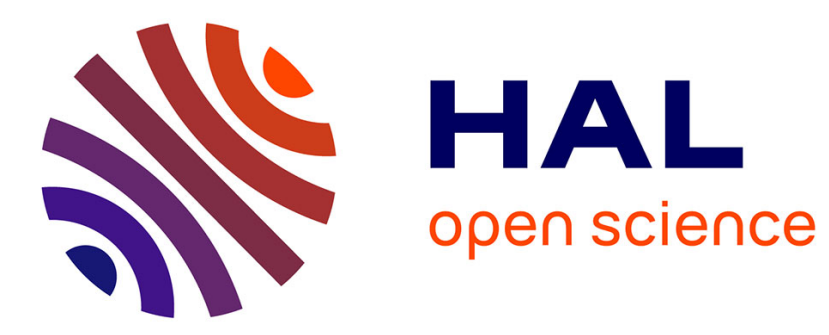

\title{
Experimental evidence of multiple stable locations for a domain wall trapped by a submicron notch
}

D Lacour, J. A Katine, L. Folks, T. Block, J. R Childress, M. J Carey, B. A Gurney

\section{- To cite this version:}

D Lacour, J. A Katine, L. Folks, T. Block, J. R Childress, et al.. Experimental evidence of multiple stable locations for a domain wall trapped by a submicron notch. Applied Physics Letters, 2004, 84 (11), pp.1910-1912. 10.1063/1.1686893 . hal-02949301

\section{HAL Id: hal-02949301 https://hal.science/hal-02949301}

Submitted on 25 Sep 2020

HAL is a multi-disciplinary open access archive for the deposit and dissemination of scientific research documents, whether they are published or not. The documents may come from teaching and research institutions in France or abroad, or from public or private research centers.
L'archive ouverte pluridisciplinaire HAL, est destinée au dépôt et à la diffusion de documents scientifiques de niveau recherche, publiés ou non, émanant des établissements d'enseignement et de recherche français ou étrangers, des laboratoires publics ou privés. 


\title{
Experimental evidence of multiple stable locations for a domain wall trapped by a submicron notch
}

\author{
D. Lacour, J. A. Katine, ${ }^{\text {a) }}$ L. Folks, T. Block, J. R. Childress, M. J. Carey, \\ and B. A. Gurney \\ Hitachi San Jose Research Center, 650 Harry Road, San Jose, California 95120
}

(Received 23 June 2003; accepted 21 January 2004)

\begin{abstract}
The presence of a domain wall trapped by a submicron notch is probed in two ways: through electronic transport measurements and by magnetic force microscopy (MFM). MFM data, exhibiting complex magnetic features, are consistent with numerical simulations predicting the existence of multiple magnetic configurations stabilized at the notch. By changing the MFM scanning history we demonstrate experimentally the existence of multiple stable locations for a domain wall trapped by a submicron notch. (C) 2004 American Institute of Physics.
\end{abstract}

[DOI: 10.1063/1.1686893]

Domain walls (DWs) play a critical role in much of the current research involving spin-dependent electronic devices. $^{1-6}$ In the case of a highly constrained DW, extremely large magnetoresistance ratios have been reported. ${ }^{1,2}$ Logic gates based on domain wall motion are being studied. ${ }^{3}$ Recently, the movement of trapped DWs via the spin-transfer torque has been experimentally demonstrated in thin films. ${ }^{4-6}$ Although the DW characteristics are key parameters in the aforementioned experiments, only a few theoretical ${ }^{7-9}$ and experimental studies ${ }^{10,11}$ have been devoted to trapped DWs.

In this letter we present an experimental study of DWs trapped by submicron notches. This study demonstrates the existence of multiple stable locations for the DWs at the notch.

Exchange biased spin-valve films consisting of $\mathrm{Ta}(3) /$ $\mathrm{NiFe}(1.5) / \operatorname{IrMn}(5) / \mathrm{CoFe}(2.5) / \mathrm{Cu}(3) / \mathrm{CoFe}(4) / \mathrm{NiFe}(15) /$ $\mathrm{Ta}(2.5)$ (thicknesses in nanometers) were sputtered onto a $\mathrm{Si}$ substrate. To fabricate the devices, we used a combination of e-beam lithography and reactive ion etching to produce a thin carbon hardmask. This mask protected the devices during the Ar ion milling used to etch the spin valve film. A second e-beam step was used to pattern thick Ta/Au leads allowing four-probe device resistance measurements to be made. The left-hand side of Fig. 1(a) is a schematic plane view of the device. There are two important features: a 50 $\mu \mathrm{m}$ square nucleation pad and a long $(100 \mu \mathrm{m})$, narrow $(0.5$ $\mu \mathrm{m})$ propagation line. The four gold leads $(I+, V+, I-$, and $V-$ ) for measuring the propagation line resistance are also shown. A rectangular notch is positioned one-third of the way along the propagation line [see Fig. 1(a) right]. Different notch geometries have been fabricated, varying the notch width $(W)$ from 500 to $50 \mathrm{~nm}$.

Typical curves of the propagation line resistance as a function of the applied field $\left(H_{\text {app }}\right)$ are shown in Fig. 1(b). The resistance measurements were performed at room temperature using a standard four-probe technique. The GMR signal is recorded during two different field loops. The first, corresponding to the $(\boldsymbol{\Delta})$ curve, is a minor loop during which

${ }^{a)}$ Electronic mail: jordan.katine@hgst.com the magnetization of the $\mathrm{CoFe} / \mathrm{NiFe}$ bilayer (called free layer in the following) is completely reversed. The minimum and maximum states of resistance correspond, respectively, to the parallel and antiparallel alignment of the spin-valve magnetizations. Intermediate plateaus at values corresponding to either $1 / 3$ or $2 / 3$ of the total resistance variation are present during the switching of the free layer magnetization. These plateaus indicate the existence of a magnetic configuration in which a DW present in the free layer is trapped at the notch. ${ }^{11}$ In order to check the stability of this configuration at remanence, the propagation line resistance has been recorded during a second field loop: the $(\bigcirc)$ curve in Fig. 1(b). After preparing the sample in a magnetic state where a DW is trapped in the notch, $H_{\text {app }}$ is reversed. Note that the resistance remains at an intermediate level for -20 $\mathrm{Oe}<\mathrm{H}_{\text {app }}<+3$ Oe. So in this field range the DW stays trapped by the notch. To further investigate this specific magnetic configuration, magnetic force microscopy (MFM) measurements have been performed.

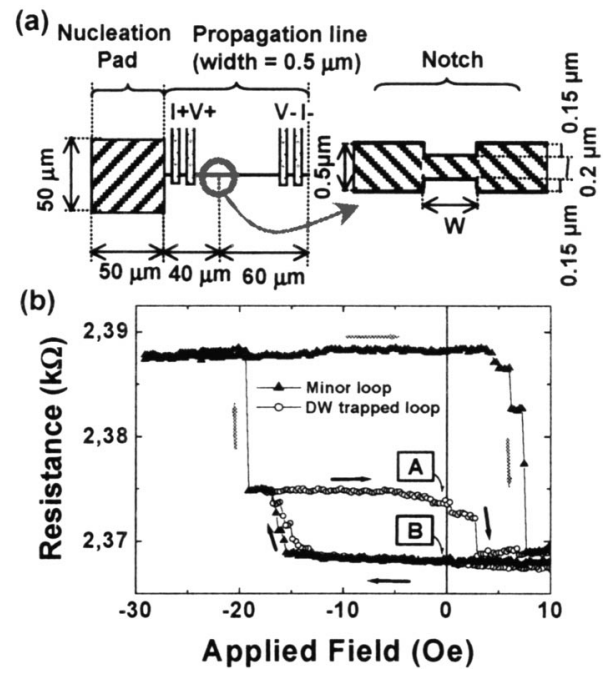

FIG. 1. (a) Schematic representation of the device. (b) Resistance as a function of the applied magnetic field recorded at room temperature for two different field loops $(\mathrm{W}=0.5 \mu \mathrm{m})$. $(\boldsymbol{\Delta})$ corresponds to a minor loop in which the magnetization of the free layer is completely switched. During the $(\bigcirc)$ loop, a DW is trapped in the notch and then stabilized in zero field. 


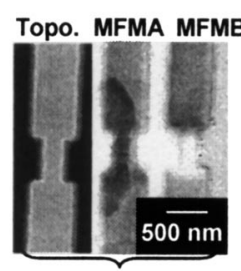

$\mathbf{W}=\mathbf{5 0 0} \mathrm{nm}$

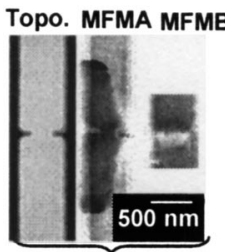

$\mathrm{W}=100 \mathrm{~nm}$

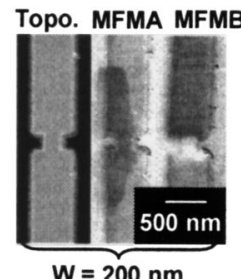

Topo. MFMA MFMB

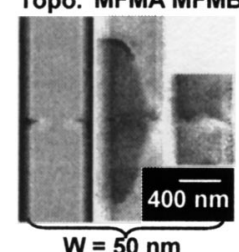

FIG. 2. Topographic (Topo) and MFM (MFMA,MFM $B$ ) pictures of notches for different geometries. The MFMA pictures correspond to the trapped DW state, while the MFM $B$ images have no DW present.

Using a commercial Digital Instruments scanning probe microscope equipped with a tip having a magnetization perpendicular to the sample surface, both topographic and MFM measurements were performed at room temperature. No external magnetic field was applied during the MFM scans, and the tip-sample separation was $18 \mathrm{~nm}$. The topographic images for four different notch shapes ( $W=500,200,100$, and $50 \mathrm{~nm}$ ) are denoted "Topo" in Fig. 2. The depth of the notch is $150 \mathrm{~nm}$ for all widths except for samples with $W=50 \mathrm{~nm}$ where proximity effects in the negative e-beam resist made the notch depth $110 \mathrm{~nm}$. With the electrical characterization technique previously described, we have been able to prepare the free layer of our samples in two different magnetic states at remanence named $A$ and $B$ in the following [see the resistance levels denoted $A$ and $B$ in Fig. 1(b)]. The state $A$ is characterized by the presence of a DW trapped by the notch. Conversely, no DW is present in state $B$. The MFM scans obtained in states $A$ and $B$, for samples with $W=500,200$, 100 , and $50 \mathrm{~nm}$, correspond, respectively, to the pictures labeled MFM $A$ and MFM $B$ in Fig. 2. Independent of the notch geometry, dark shapes (resembling a bat) centered on the notch are present on the MFMA pictures, while two black and white areas surrounding the notch are observable in the MFM $B$ pictures.

The contrast of MFM $B$ images have been reproduced well using the micromagnetic model OOMMF ${ }^{12}$ (not shown here). Using the same model, the micromagnetic configuration and resulting MFM image at remanence has also been computed in the case of a trapped DW (state $B$ ) for different reverse fields values. Figure 3 shows the results of these simulations. The value of the reverse field is decreased in 20 Oe steps, starting from -60 Oe for simulation (i) and ending at -120 Oe for simulation (iv). Note that the effect of temperature is not taken into account in these simulations, leading to an overestimation of the propagation field values. For a comparison with MFM pictures the magnetic pole density: $\rho=-\operatorname{div} M$ (leading to appearance of the MFM contrast) is represented by a gray scale in the simulated pictures. Depending on the reverse field value, different wall shapes and wall positions can be attained at remanence. So the simulaDownloaded 23 Mar 2004 to 64.254.97.10. Redistribution subject to (i)

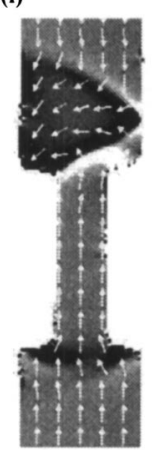

(ii)

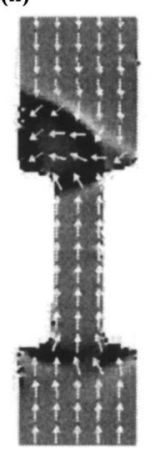

(iii)

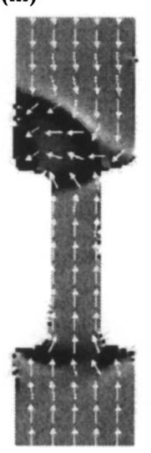

(iv)

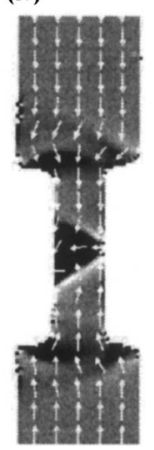

FIG. 3. Micromagnetic simulations of a trapped DW at remanence for different reverse field values. The gray scale represents the magnetic pole density. The values of the reverse fields are, respectively, equal to -60 , $-80,-100,-120$ Oe for the simulations (i), (ii), (iii), and (iv).

tions predict the existence of multiple possible trapped DW positions at remanence. But none of these simulations produces MFM images similar to the observed "bat" shape which our magnetoresistance measurements unambiguously associate with the presence of a trapped DW. Note, however, that the observed "bat" shape may be obtained via a superposition of the different simulated states and of their mirrors. This strongly suggests that the DW is imaged in different positions during the same MFM scan. Hence, the observed "bat" shape likely originates from DW motion induced by the tip. Such motion is not surprising. The effect of MFM tips on micromagnetic configuration was widely studied when the technique was introduced; specifically, DW motion effects induced by MFM tips were observed by Mamin et $a l .{ }^{13}$ In order to confirm this hypothesis we have performed MFM scans in which we vary the scanning history.

Figure 4 shows the results obtained when a DW is present in the free layer for devices with $W=500,200,100$ nm. The smaller notch case $(W=50 \mathrm{~nm})$ will be discussed later. Three different scanning procedures have been used; they are named (a), (b), and (c) in the following. Each of them includes two successive scans and the pictures are always recorded during the second scan. The procedure (a) consists in a scan starting from the "bottom" of the device and ending at the "top" of the element immediately followed by a scan performed in the opposite direction (starting from the "top"). During procedure (b) the two successive scans are performed in the same direction from the "bottom" to the "top" part of the device. For procedure (c) the two successive scans are performed from the "top" to the "bottom." The global trajectory followed by the tip during each procedure is noted by arrows in Fig. 4. The MFM pictures re-

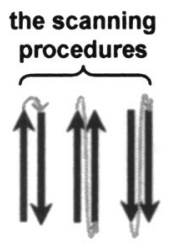

(a) (b) (c)

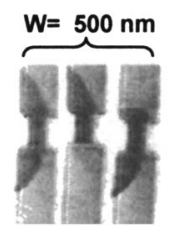

(a) (b) (c)

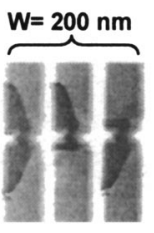

(a) (b) (c)

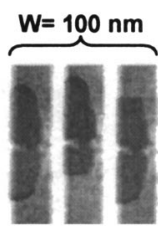

(a) (b) (c)

FIG. 4. Schematic representation of the three scanning procedures and MFM pictures of the trapped DW configurations recorded in using these procedures. 
corded using procedures (a), (b), and (c) are labeled, respectively, (a), (b), and (c) in Fig. 4. If procedure (a) leads to the observation of the full "bat" shape as expected, ${ }^{14}$ procedures (b) and (c) generate a discontinuity during the magnetic signal recording. Only the top (bottom) wing of the bat is present in images when procedure (b) [procedure (c)] is performed. These three behaviors can be easily understood by taking the DW motion induced by the MFM tip into account. During the first scan of procedure (a), the tip leaves the DW in a position bordering the top of the notch, and then during the second scan, the tip, moving from top-to-bottom, displaces the DW through the notch. So the DW is imaged in all of the trapping positions induced by the notch, and this leads to the complete bat observation. At the end of the first scan of procedure (b), the tip leaves the DW in a position bordering the top of the notch. During the second scan, the MFM tip is unable to image the DW in the bottom position, simply because the domain wall is situated in the top position. So the bottom wing of the bat is missing. When the tip approaches close enough to the domain wall, the wall is attracted in an irreversible way, and this leads to the discontinuity in the magnetic signal observed in (b). Then the DW experiences the trapping positions situated on the tip trajectory. This leads to the imaging of the top part of the bat. If the two scanning directions of procedure (b) are reversed, we expect that the top wing of the bat will be missing, as observed in the images recorded using procedure (c). The same experiments were performed on the smallest notch devices $(W=50 \mathrm{~nm})$, but the results obtained were not reproducible from one device to another. We believe that this is due to weaker pinning in the shallower notch depth realized at this dimension.

In conclusion, stripe shaped spin-valves including a submicron notch have been fabricated in order to investigate trapped DWs properties. Combining micromagnetic simulations, transport, and an original MFM measurement technique, we have demonstrated that multiple stable locations for DWs are created at the notch. Furthermore, the shape and the stability of the wall appears to be dependent on the trapping position. These results should be taken into account when dealing with spin-dependent electronic devices involving trapped DWs because the magneto-electronic properties can change drastically from one stable position to another.

The authors wish to acknowledge M. Hehn and Z. Z. Bandić for helpful discussions.

${ }^{1}$ G. Tatara, Y.-W. Zhao, M. Munoz, and N. Garcia, Phys. Rev. Lett. 83, 2030 (1999).

${ }^{2}$ H. D. Chopra and S. Z. Hua, Phys. Rev. B 66, 020403 (2002).

${ }^{3}$ D. A. Allwood, G. Xiong, M. D. Cooke, C. C. Faulkner, D. Atkinson, N. Vernier, and R. P. Cowburn, Science 296, 2003 (2002).

${ }^{4}$ N. Vernier, D. A. Allwood, D. Atkinson, M. D. Cooke, and R. P. Cowburn, Europhys. Lett. 65, 526 (2004).

${ }^{5}$ J. Grollier, D. Lacour, V. Cros, A. Hamzic, A. Vaurès, A. Fert, D. Adam, and G. Faini, J. Appl. Phys. 92, 4825 (2002).

${ }^{6} \mathrm{~J}$. Grollier, P. Boulenc, V. Cros, A. Hamzic, A. Vaurès, A. Fert, and G. Faini, Appl. Phys. Lett. 83, 509 (2003).

${ }^{7}$ P. Bruno, Phys. Rev. Lett. 83, 2425 (1999).

${ }^{8}$ R. D. McMichael, J. Eicke, M. J. Donahue, and D. G. Porter, J. Appl. Phys. 87, 7058 (2000).

${ }^{9}$ V. A. Molyneux, V. V. Osipov, and E. V. Ponizovskaya, Phys. Rev. B 65, 184425 (2002).

${ }^{10}$ M. Klaui, C. A. F. Vaz, J. Rothman, J. A. C. Bland, W. Wernsdorfer, G. Faini, and E. Cambril, Phys. Rev. Lett. 90, 097202 (2003).

${ }^{11}$ T. Ono, H. Miyajima, K. Shigeto, T. Shigeto, and T. Shinjo, Appl. Phys. Lett. 72, 1116 (1998).

${ }^{12} \mathrm{http}: / /$ math.nist.gov/oommf.

${ }^{13}$ H. J. Mamin, D. Rugar, J. E. Stern, R. E. Fontana, and P. Kasiraj, Appl. Phys. Lett. 55, 318 (1989).

${ }^{14}$ Indeed, we have used procedure (a) for the acquisition of pictures shown in Fig. 2. Note that (a) is the default procedure currently used in scanned probe microscopy. 